\title{
New and rapid access to synthesis of novel polysubstituted imidazoles using antimony trichloride and stannous chloride dihydrate as effective and reusable catalysts
}

\author{
LEYLA POORALI $^{\mathrm{a}}$, BAHADOR KARAMI $^{\mathrm{b}, *}$, KHALIL ESKANDARI $^{\mathrm{b}}$ \\ and MAHBOOBEH AZIZI ${ }^{\mathrm{a}}$ \\ a Department of Chemistry, Gachsaran Branch, Islamic Azad University, Gachsaran, 75817-75785, Iran \\ bepartment of Chemistry, Yasouj University, Yasouj, 75918-74831 P.O. Box 353, Iran \\ e-mail:karami@mail.yu.ac.ir
}

MS received 12 March 2012; revised 8 October 2012; accepted 17 October 2012

\begin{abstract}
In this work, new, efficient and environmentally adapted synthesis of polysubstituted imidazoles in one-pot is repoted. The multicomponent reaction of various aldehydes, benzil, aliphatic and aromatic primary amines and ammonium acetate under solvent-free condition is explained. The highly efficient role of antimony trichloride and stannous chloride dihydrate as catalyst in this synthesis was shown and their effects on the reaction process were studied. By this advantage, several polysubstituted imidazoles as pharmaceutical important molecules can be prepared in high yield and high purity. This method is a very easy and rapid for the synthesis of imidazole derivatives.
\end{abstract}

Keywords. Benzil; polysubstituted imidazoles; aldehydes; amines; heterogeneous catalyst.

\section{Introduction}

Multicomponent reactions (MCRs) are the special type of organic reactions which afford complex products from reaction of three or more simple starting materials in one pot. Because of atom-economy, convergent character, operational simplicity, structural diversity and complexity of the molecules in these reactions, MCR's have attracted much attention. ${ }^{1,2}$

The imidazoles and their derivatives are very important molecules because they have many applications in chemical processes, especially in pharmaceuticals. ${ }^{3,4}$ Various substituted imidazoles act as inhibitors of p38 MAP kinase, ${ }^{5}$ B-Raf kinase, ${ }^{6}$ glucagon receptors, ${ }^{7}$ plant growth regulators, ${ }^{8}$ antitumour ${ }^{9}$ and pesticides. ${ }^{10}$

Solid and Lewis acid catalysts have been of great interest in organic synthesis. ${ }^{11}$ There are many methods for the synthesis of polysubstituted imidazoles by employing of Lewis acids ${ }^{12}$ such as condensation of diones, aldehydes, primary amines and ammonia in the presence of various acid catalysts, ${ }^{13-15} \mathrm{~N}$-alkylation of trisubstituted imidazoles, ${ }^{16}$ condensation of benzil or

*For correspondence benzoin acetate with aldehydes, primary amines and ammonia in the presence of copper acetate, ${ }^{17,18}$ condensation of $o$-diamines with aldehydes and in the presence of tetrabutylammonium fluoride (TBAF), ${ }^{19}$ etc. The first method is the most well-known and classical method. However, some of these methods, involved long reaction times, and unsatisfactory yields. Therefore, improvements in these syntheses have been sought continuously. ${ }^{20}$ In the scope of previous works for synthesis of heterocyclic compounds by inorganic solid acids, ${ }^{21}$ in this study, a new method for the synthesis of polysubstituted imidazoles was obtained by condensation of benzil with aldehydes, primary amines and ammonium acetate in the presence of antimony trichloride and stannous chloride dihydrate as effective and reusable catalysts in solvent-free condition (scheme 1). Here, we report a simple, rapid and one-pot procedure for the synthesis of tri and tetrasubstituted imidazoles by the use of antimony trichloride and stannous chloride dihydrate with high yields and short reaction times. Using benzil 1, aromatic aldehydes 2, aromatic and aliphatic amine 3, ammonium acetate $\mathbf{4}$ and catalytic amount of $\mathrm{SbCl}_{3}$ (or $\mathrm{SnCl}_{2} \cdot 2 \mathrm{H}_{2} \mathrm{O}$ ) under solventfree condition lead to tetrasubstituted imidazoles $\mathbf{5}$, wherease in the absence of aromatic and aliphatic amine 3, trisubstituted imidazoles were obtained $\mathbf{5}^{\prime}$ (see scheme 1). 


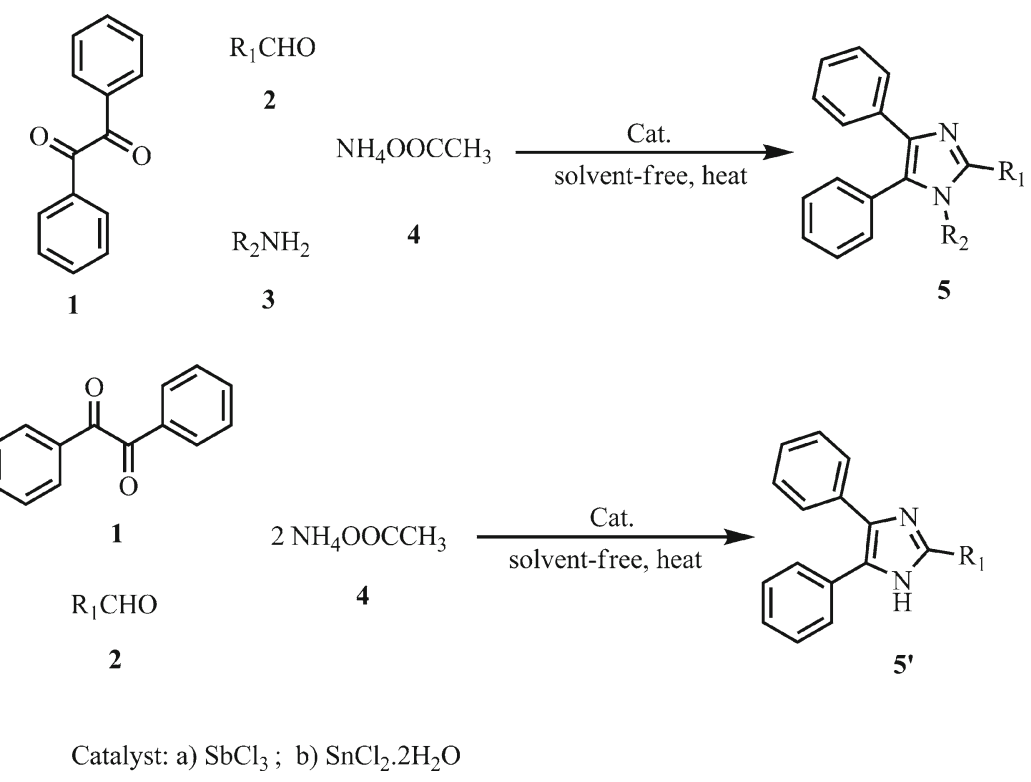

Scheme 1. Synthesis of polysubstituted imidazoles by the use of $\mathrm{SbCl}_{3}$ and $\mathrm{SnCl}_{2} \cdot 2 \mathrm{H}_{2} \mathrm{O}$ as catalyst.

\section{Experimental}

Melting points were measured on an elecrtothermal KSB1N apparatus. IR spectra were recorded in the matrix of $\mathrm{KBr}$ with JASCO FT-IR-680 plus spectrometer. ${ }^{1} \mathrm{H}$ NMR and ${ }^{13} \mathrm{C}$ NMR spectra were recorded on a FT-NMR Bruker Avance ultra shield spectrometer at 400.13 and $100.62 \mathrm{MHz}$ in $\mathrm{CDCl}_{3}$ and $\mathrm{DMSO}-\mathrm{d}_{6}$ as solvent in the presence of tetramethylsilane as internal standard. TLC was performed on TLC-Grade silica gel-G/UV $254 \mathrm{~nm}$ plates. The products were isolated and characterized by physical and spectral data and they were compared with authentic samples (table 1).

\subsection{General procedure for synthesis of}

\section{1,2,4,5-tetrasubstituted imidazoles by $\mathrm{SbCl}_{3}$} and $\mathrm{SnCl}_{2} \cdot 2 \mathrm{H}_{2} \mathrm{O}$

A mixture of aromatic aldehyde $(1 \mathrm{mmol})$, benzil $(1 \mathrm{mmol})$, primary amine $(1 \mathrm{mmol})$, ammonium acetate $(1 \mathrm{mmol})$ and $\mathrm{SbCl}_{3}(5 \mathrm{~mol} \%)$ (for $\mathrm{SnCl}_{2} \cdot 2 \mathrm{H}_{2} \mathrm{O}$ $20 \mathrm{~mol} \%$ ) were stirred at $120^{\circ} \mathrm{C}$ (when $\mathrm{SnCl}_{2} \cdot 2 \mathrm{H}_{2} \mathrm{O}$ handling as catalyst at $140^{\circ} \mathrm{C}$ ) in solvent-free condition. The progress of reaction was monitored by TLC. After completion of reaction, the mixture was cooled to room temperature and was solved in $50 \mathrm{~mL}$ water then was filtered. Obtained products were purified by crystallization from acetone-water (10:1). The products were characterized by IR, NMR, and through comparison of their physical properties with those reported in literature. ${ }^{15,22-32}$ Hence, general procedure for the synthesis of 2,4,5-trisubstituted imidazoles is same to the 1,2,4,5-tetrasubstituted imidazoles, but it needs $2 \mathrm{mmol}$ of ammonium acetate instead of $1 \mathrm{mmol}$ in the absence of primary amine.

\subsection{Representative spectral data}

2.2a Compound 5a (table 1): m.p. $170-172^{\circ} \mathrm{C}$; Yield $0.360 \mathrm{~g}, 96 \%$; IR $(\mathrm{KBr})\left(v_{\max }, \mathrm{cm}^{-1}\right): 3061$, 3026, 2308, 1601, 1521, 1497, 1350, 761, 696. ${ }^{1} \mathrm{H}$ NMR $(250 \mathrm{MHz}$, DMSO-d6): $\delta(\mathrm{ppm}) 5.13(\mathrm{~s}, 2 \mathrm{H})$, 7.18-8.41(m, 20H). ${ }^{13} \mathrm{CNMR}\left(62.69 \mathrm{MHz}, \mathrm{DMSO}_{-} \mathrm{d}_{6}\right)$ $\delta$ (ppm): 48.2, 126.0, 126.3, 126.8, 128.1, 129.0, 129.5, $129.9,130.9,130.9,131.0,135.0,137.5,148.0$.

2.2b Compound 5b (table 1): m.p. $169-170^{\circ} \mathrm{C}$; Yield $0.418 \mathrm{~g}, 90 \%$; IR (KBr) $\left(v_{\max }, \mathrm{cm}^{-1}\right): 3059,3027$, 2938, 1599, 1479, 1358, 1070, 835, 758, 694. ${ }^{1} \mathrm{H}$ NMR (400.13 MHz, DMSO-d6): $\delta(\mathrm{ppm}) 4.86$ (s, 2H), 6.61$7.46(\mathrm{~m}, 19 \mathrm{H}) .{ }^{13} \mathrm{CNMR}\left(100.62 \mathrm{MHz}, \mathrm{DMSO}-\mathrm{d}_{6}\right) \delta$ (ppm): 49.4, 125.8, 126.5, 126.7, 127.5, 128.1, 128.2, 128.7, 128.8, 130.4, 130.7, 131.0, 131.7, 137.3.

2.2c Compound 5c (table 1): m.p. $163-166^{\circ} \mathrm{C}$; Yield $0.368 \mathrm{~g}, 92 \%$; IR $(\mathrm{KBr})\left(v_{\max }, \mathrm{cm}^{-1}\right): 3060$, 3027, 2926, 1600, 1496, 1349, 826, 767, 694. ${ }^{1} \mathrm{HNMR}$ (400.13 MHz, DMSO-d $\left.{ }_{6}\right) \delta$ (ppm): 2.08 (s, 3H), 4.91 (s, 2H), 6.61-7.40 (m, 19H). ${ }^{13} \mathrm{CNMR}(100.62 \mathrm{MHz}$, DMSO-d $\left._{6}\right) \delta$ (ppm): 22.5, 49.4, 127.1, 127.4, 127.9, $128.4,129.2,129.7,129.9,130.1,130.4,131.0,132.2$, $138.8,140.0,146.3$. 
Table 1. Synthesis of polysubstituted imidazoles catalysed by $\mathrm{SbCl}_{3}$ and $\mathrm{SnCl}_{2} \cdot 2 \mathrm{H}_{2} \mathrm{O}$ under solvent-free condition.

\begin{tabular}{|c|c|c|c|c|c|}
\hline & & & $\mathrm{SnCl}_{2} \cdot 2 \mathrm{H}_{2} \mathrm{O}$ & $\mathrm{SbCl}_{3}$ & \\
\hline Compound & $\mathrm{R}^{1}$ & $\mathrm{R}^{2} \mathrm{NH}_{2}$ & $\begin{array}{c}\text { Time/Yield } \\
(\mathrm{min}) /(\%)\end{array}$ & $\begin{array}{c}\text { Time/Yield }^{\mathrm{a}} \\
(\mathrm{min}) /(\%)\end{array}$ & $\begin{array}{c}\text { M.p. } \\
\left({ }^{\circ} \mathrm{C}\right) /(\text { lit. })\end{array}$ \\
\hline $5 \mathbf{a}$ & $\mathrm{C}_{6} \mathrm{H}_{5}$ & $\mathrm{C}_{6} \mathrm{H}_{5} \mathrm{CH}_{2} \mathrm{NH}_{2}$ & $50 / 96$ & $30 / 96$ & $170-172^{15}$ \\
\hline $5 \mathbf{b}$ & $4-\mathrm{Br}-\mathrm{C}_{6} \mathrm{H}_{4}$ & $\mathrm{C}_{6} \mathrm{H}_{5} \mathrm{CH}_{2} \mathrm{NH}_{2}$ & $25 / 92$ & $20 / 90$ & $169-170^{15}$ \\
\hline $5 c$ & 4- $-\mathrm{CH}_{3}-\mathrm{C}_{6} \mathrm{H}_{4}$ & $\mathrm{C}_{6} \mathrm{H}_{5} \mathrm{CH}_{2} \mathrm{NH}_{2}$ & $80 / 98$ & $55 / 92$ & $163-166^{22}$ \\
\hline $5 d$ & $4-\mathrm{Cl}-\mathrm{C}_{6} \mathrm{H}_{4}$ & $\mathrm{C}_{6} \mathrm{H}_{5} \mathrm{CH}_{2} \mathrm{NH}_{2}$ & $60 / 90$ & $25 / 92$ & $160-162^{22}$ \\
\hline $5 e$ & $2-\mathrm{Cl}-\mathrm{C}_{6} \mathrm{H}_{4}$ & $\mathrm{C}_{6} \mathrm{H}_{5} \mathrm{CH}_{2} \mathrm{NH}_{2}$ & $20 / 88$ & $20 / 90$ & $193-195^{23}$ \\
\hline $5 f$ & $3-\mathrm{NO}_{2}-\mathrm{C}_{6} \mathrm{H}_{4}$ & $\mathrm{C}_{6} \mathrm{H}_{5} \mathrm{CH}_{2} \mathrm{NH}_{2}$ & $40 / 85$ & $30 / 90$ & $150-152^{\mathrm{b}}$ \\
\hline $5 g$ & 4- $-\mathrm{CH}_{3}-\mathrm{C}_{6} \mathrm{H}_{4}$ & CyclohexylNH $_{2}$ & $40 / 75$ & $30 / 88$ & $160-161^{24}$ \\
\hline $5 \mathrm{~h}$ & $4-\mathrm{OCH}_{3}-\mathrm{C}_{6} \mathrm{H}_{4}$ & $\mathrm{C}_{6} \mathrm{H}_{5} \mathrm{CH}_{2} \mathrm{NH}_{2}$ & $60 / 88$ & $50 / 90$ & $148-151^{25}$ \\
\hline $5 i$ & $2-\mathrm{OH}-5-\mathrm{Br}-\mathrm{C}_{6} \mathrm{H}_{3}$ & $4-\mathrm{Cl}-\mathrm{C}_{6} \mathrm{H}_{4} \mathrm{NH}_{2}$ & $30 / 92$ & $25 / 98$ & $156-158^{\mathrm{b}}$ \\
\hline $5 \mathbf{j}$ & 4-Benzyloxy- $\mathrm{C}_{6} \mathrm{H}_{4}$ & $\mathrm{C}_{6} \mathrm{H}_{5} \mathrm{CH}_{2} \mathrm{NH}_{2}$ & $80 / 85$ & $80 / 96$ & $138-139^{\mathrm{b}}$ \\
\hline $5 \mathbf{k}$ & 2,4-di-Cl- $\mathrm{C}_{6} \mathrm{H}_{3}$ & $\mathrm{C}_{6} \mathrm{H}_{5} \mathrm{CH}_{2} \mathrm{NH}_{2}$ & $85 / 80$ & $70 / 90$ & $216-219^{\mathrm{b}}$ \\
\hline 51 & $4-\mathrm{CH}_{3}-\mathrm{C}_{6} \mathrm{H}_{4}$ & $\mathrm{C}_{6} \mathrm{H}_{5} \mathrm{NH}_{2}$ & $60 / 88$ & $30 / 90$ & $182-184^{26}$ \\
\hline $5^{\prime} \mathbf{a}$ & $\mathrm{C}_{6} \mathrm{H}_{5}$ & -- & $50 / 90$ & $15 / 95$ & $272-273^{27}$ \\
\hline $5^{\prime} \mathbf{b}$ & $3-\mathrm{Br}-\mathrm{C}_{6} \mathrm{H}_{4}$ & --- & $30 / 85$ & $10 / 88$ & $120-122^{28}$ \\
\hline $5^{\prime} \mathrm{c}$ & 2-OH- $\mathrm{C}_{6} \mathrm{H}_{4}$ & --- & $60 / 88$ & $15 / 90$ & $209-211^{29}$ \\
\hline $5^{\prime} d$ & $2-\mathrm{OCH}_{3}-\mathrm{C}_{6} \mathrm{H}_{4}$ & --- & $60 / 90$ & $25 / 92$ & $204-206^{25}$ \\
\hline $5^{\prime} \mathbf{e}$ & $4-\mathrm{OCH}_{3}-\mathrm{C}_{6} \mathrm{H}_{4}$ & --- & $45 / 85$ & $15 / 88$ & $227-230^{27}$ \\
\hline $5^{\prime} f$ & 4-Benzyloxy- $\mathrm{C}_{6} \mathrm{H}_{4}$ & --- & $45 / 75$ & $20 / 80$ & $235-236^{30}$ \\
\hline $5^{\prime} \mathrm{g}$ & 2-Fluorenyle & --- & $50 / 90$ & $25 / 97$ & $283-286^{\mathrm{b}}$ \\
\hline $5^{\prime} \mathbf{h}$ & 3-Indolyl & --- & $60 / 80$ & $30 / 88$ & $311-313^{\mathrm{b}}$ \\
\hline $5^{\prime} \mathbf{i}$ & 4- $\mathrm{Cl}-\mathrm{C}_{6} \mathrm{H}_{4}$ & --- & $40 / 90$ & $10 / 94$ & $257-259^{31}$ \\
\hline $5^{\prime} \mathbf{j}$ & $3-\mathrm{NO}_{2}-\mathrm{C}_{6} \mathrm{H}_{4}$ & --- & $45 / 90$ & $10 / 92$ & $308-309^{32}$ \\
\hline
\end{tabular}

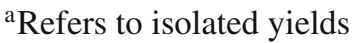

bovel compound

2.2d Compound 5d (table 1): m.p. $160-162^{\circ} \mathrm{C}$; Yield 0.386 g, 92\%; IR (KBr) $\left(v_{\max }, \mathrm{cm}^{-1}\right): 3059,3029$, 2936, 1600, 1480, 1357, 1089, 835, 758, 693. ${ }^{1} \mathrm{HNMR}$ (400.13 MHz, DMSO-d $\left.{ }_{6}\right) \delta(\mathrm{ppm}): 4.9$ (s, 2H), 6.60$7.50(\mathrm{~m}, 19 \mathrm{H}) .{ }^{13} \mathrm{CNMR}(100.62 \mathrm{MHz}$, DMSO-d 6$) \delta$ (ppm): 48.3, 125.8, 126.5, 127.5, 129.1, 129.3, 130.2, 130.4, 130.7, 131.0, 134.1, 135.0, 136.8, 137.3, 138.2.

2.2e Compound 5e (table 1): m.p. $193-195^{\circ} \mathrm{C}$; Yield $0.378 \mathrm{~g}, 90 \%$; IR $(\mathrm{KBr})\left(v_{\max }, \mathrm{cm}^{-1}\right): 3062,3026$, 2930, 1602, 1485, 1349, 1079, 758, 690. ${ }^{1} \mathrm{HNMR}$ $\left(400.13 \mathrm{MHz}, \mathrm{DMSO}-\mathrm{d}_{6}\right) \delta(\mathrm{ppm}): 4.74$ (s, 2H), 6.42$7.42(\mathrm{~m}, 19 \mathrm{H}) .{ }^{13} \mathrm{CNMR}\left(100.62 \mathrm{MHz}, \mathrm{DMSO}-\mathrm{d}_{6}\right) \delta$ (ppm): 43.1, 126.0, 126.5, 126.6, 127.4, 128.4, 128.6, $128.9,129.1,129.3,129.7,129.8,130.3,130.6,130.8$, 131.0, 131.1, 132.9, 133.8, 134.2, 134.9, 136.8, 137.6, 145.1.

2.2f Compound $\mathbf{5 f}($ table 1$)$ : $\quad$ m.p. $150-152^{\circ} \mathrm{C}$; Yield $0.388 \mathrm{~g}, 90 \%$; IR $(\mathrm{KBr})\left(v_{\max }, \mathrm{cm}^{-1}\right): 3061,3026,2308$, 1601, 1521, 1497, 1350, 810, 730, 696. ${ }^{1} \mathrm{H}$ NMR (400.13 MHz, DMSO-d6): $\delta$ (ppm): 5.19 (s, 2H), 6.89 $(\mathrm{d}, J=6.15 \mathrm{~Hz}, 2 \mathrm{H}), 7.21-7.63(\mathrm{~m}, 14 \mathrm{H}), 8.04(\mathrm{~d}$,
$J=7.8 \mathrm{~Hz}, 1 \mathrm{H}), 8.23(\mathrm{~d}, J=7.8 \mathrm{~Hz}, 1 \mathrm{H}), 8.57(\mathrm{~s}$, 1H). ${ }^{13}$ CNMR (100.62 MHz, DMSO-d 6 ) $\delta$ (ppm): 47.4, 122.3, 122.5, 124.7, 125.6, 125.7, 126.7, 127.1, 127.8, $127.9,128.5,129.3,129.9,130.2,131.5,132.9,133.5$, 135.7, 144.2, 147.2. m/z: $431 \mathrm{M}^{+}, 386,340,295,190$, 165, 134, 91, 57. Anal. Calcd for $\mathrm{C}_{28} \mathrm{H}_{21} \mathrm{~N}_{3} \mathrm{O}_{2}$ : C, 77.94; H, 4.91; N, 9.74. Found: C, 77.87; H, 4.83; N, 9.62.

$2.2 \mathrm{~g}$ Compound 5g (table 1): m.p. $160-161^{\circ} \mathrm{C}$; Yield $0.345 \mathrm{~g}, 88 \%$; IR $(\mathrm{KBr})\left(v_{\max }, \mathrm{cm}^{-1}\right): 3058$, 3020, 2930, 1600, 1495, 1349, 825, 766, 695. ${ }^{1} \mathrm{HNMR}$ (400.13 MHz, $\left.\mathrm{CDCl}_{3}\right) \delta(\mathrm{ppm}): 0.95(\mathrm{~m}, 2 \mathrm{H}), 1.35-1.55$ (m, 6H), $1.75(\mathrm{~m}, 2 \mathrm{H}), 2.35$ (s, 3H), 3.80-3.97 (m, 1H), 6.70-7.45 (m, 14H). ${ }^{13} \mathrm{CNMR}\left(100.62 \mathrm{MHz}, \mathrm{CDCl}_{3}\right) \delta$ (ppm): 21.4, 25.1, 26.2, 33.5, 58.3, 125.9, 126.0, 126.6, 127.6, 127.8, 127.9, 128.1, 128.6, 128.7, 128.8, 129.0, 129.0, 129.1, 129.5, 129.8, 132.2, 132.8, 134.7, 137.6, 138.7, 147.8 .

2.2h Compound 5h (table 1): m.p. $148-151^{\circ} \mathrm{C}$; Yield $0.374 \mathrm{~g}, 90 \%$; IR $(\mathrm{KBr})\left(v_{\max }, \mathrm{cm}^{-1}\right)$ : 3063, 3019, 2295, 1609, 1519, 1493, 1354, 696. ${ }^{1} \mathrm{H}$ NMR $\left(400.13 \mathrm{MHz}, \mathrm{CDCl}_{3}\right): \delta(\mathrm{ppm}) 3.81(\mathrm{~s}, 3 \mathrm{H}), 5.08(\mathrm{~s}$, 
$2 \mathrm{H}), 6.82-7.56(\mathrm{~m}, 15 \mathrm{H}), 7.58(\mathrm{~d}, J=2.3 \mathrm{~Hz}, 4 \mathrm{H})$. ${ }^{13} \mathrm{CNMR}\left(100.62 \mathrm{MHz}, \mathrm{CDCl}_{3}\right) \delta$ (ppm): 47.1, 54.2, $112.9,122.4,124.9,125.2,125.7,126.2,127.0,127.4$, $127.5,127.7,128.0,128.7,129.3,130.0,130.1,133.5$, 136.6, 136.7, 146.9, 159.0.

2.2i Compound 5i (table 1): m.p. $156-158^{\circ} \mathrm{C}$; Yield $0.490 \mathrm{~g}, 98 \%$; IR (KBr) $\left(v_{\max }, \mathrm{cm}^{-1}\right): 3458,3063,1659$, 1593, 1578, 1211, 1174, 1096. ${ }^{1} \mathrm{HNMR}(400.13 \mathrm{MHz}$, DMSO-d $\left._{6}\right) \delta(\mathrm{ppm}): 6.92-8.53(\mathrm{~m}, 17 \mathrm{H}), 13.06(\mathrm{~s}, 1 \mathrm{H})$. ${ }^{13} \mathrm{CNMR}\left(100.62 \mathrm{MHz}, \mathrm{DMSO}-\mathrm{d}_{6}\right) \delta(\mathrm{ppm}): 115.1$, 123.9, 125.5, 127.6, 134.3, 137.3, 139.2, 140.5, 151.3, 164.7, 166.9. m/z: 502 M+, 267, 214, 193, 165, 75, 57. Anal. Calcd for $\mathrm{C}_{27} \mathrm{H}_{18} \mathrm{BrClN}_{2} \mathrm{O}: \mathrm{C}, 64.62 ; \mathrm{H}, 3.62 ; \mathrm{N}$, 5.58. Found: C, 64.51; H, 3.53; N, 5.49.

2.2j Compound $\mathbf{5 j}$ (table 1): m.p. $138-139^{\circ} \mathrm{C}$; Yield $0.472 \mathrm{~g}, 96 \%$; IR (KBr) $\left(v_{\max }, \mathrm{cm}^{-1}\right): 2857,1601$, 1575, 1526, 1289, 1247, 1177. ${ }^{1} \mathrm{HNMR}(400.13 \mathrm{MHz}$, DMSO-d $\left._{6}\right) \delta(\mathrm{ppm}): 2.18(\mathrm{~s}, 2 \mathrm{H}), 5.06(\mathrm{~s}, 2 \mathrm{H}), 6.83-$ $7.82(\mathrm{~m}, 24 \mathrm{H}) .{ }^{13} \mathrm{CNMR}\left(100.62 \mathrm{MHz}, \mathrm{DMSO}_{-}\right) \delta$ (ppm): 48.2, 70.0, 115.0, 123.7, 126.0, 126.3, 126.8, 127.4, 127.5, 128.1, 128.1, 128.6, 128.8, 129.8, 130.5, $131.1,131.2,134.7,136.7,137.7,137.9,148.0,159.3$. m/z: $492 \mathrm{M}^{+}$, 402, 311, 283, 165, 91, 65. Anal. Calcd for $\mathrm{C}_{35} \mathrm{H}_{28} \mathrm{~N}_{2} \mathrm{O}$ : C, 85.34; H, 5.73; N, 5.69. Found: C, 85.18; H, 5.61; N, 5.54.

$2.2 \mathrm{k}$ Compound 5k (table 1): m.p. $216-219^{\circ} \mathrm{C}$; Yield $0.407 \mathrm{~g}, 90 \%$; IR $(\mathrm{KBr})\left(v_{\max }, \mathrm{cm}^{-1}\right): 3069$, 2924, 1557, 1523, 1459, 1092. ${ }^{1}$ HNMR (400.13 MHz, DMSO-d $\left._{6}\right) \delta$ (ppm): 2.18 (s, 2H), 7.45-8.41 (m, 13H), $8.64(\mathrm{~d}, J=7.6 \mathrm{~Hz}, 1 \mathrm{H}), 8.77(\mathrm{t}, J=8.4 \mathrm{~Hz}, 2 \mathrm{H})$. ${ }^{13} \mathrm{CNMR}$ (100.62 MHz, DMSO-d $\left.\mathrm{d}_{6}\right) \delta$ (ppm): 120.8, 121.1, 122.9, 123.4, 123.7, 125.0, 126.0, 126.8, 127.2, 127.3, 127.4, 127.5, 129.0, 129.5, 131.2, 132.3, 133.7, 135.2, 136.9, 145.2. $\mathrm{m} / \mathrm{z}: 452 \mathrm{M}^{+}, 363,295,190,164$, 91. Anal. Calcd for $\mathrm{C}_{28} \mathrm{H}_{20} \mathrm{Cl}_{2} \mathrm{~N}_{2}: \mathrm{C}, 73.85 ; \mathrm{H}, 4.43 ; \mathrm{N}$, 6.15. Found: C, 73.71; H, 4.36; N, 6.09.

2.21 Compound $\mathbf{5 l}$ (table 1): m.p. $182-184^{\circ} \mathrm{C}$; Yield $0.347 \mathrm{~g}, 90 \%$; IR (KBr) $\left(v_{\max }, \mathrm{cm}^{-1}\right): 3055,3022$, 2930, 1600, 1495, 1349, 826, 767, 694. ${ }^{1} \mathrm{H}$ NMR $\left(400.13 \mathrm{MHz}, \mathrm{CDCl}_{3}\right): \delta(\mathrm{ppm}) 2.34(\mathrm{~s}, 3 \mathrm{H}), 7.07-$ $7.36(\mathrm{~m}, 17 \mathrm{H}), 7.64(\mathrm{~d}, J=7.1 \mathrm{~Hz}, 2 \mathrm{H}) .{ }^{13} \mathrm{CNMR}$ $\left(100.62 \mathrm{MHz}, \mathrm{CDCl}_{3}\right) \delta$ (ppm): 20.2, 124.2, 125.4, 126.3, 126.6, 126.8, 127.0, 127.1, 127.2, 127.4, 127.7, $127.7,127.9$, 128.4, 129.7, 130.1, 136.2, 137.1, 146.0.

$2.2 \mathrm{~m}$ Compound $\mathbf{5}^{\prime} \mathbf{b}$ (table 1): m.p. $120-122^{\circ} \mathrm{C}$; Yield $0.330 \mathrm{~g}, 88 \%$; IR (KBr) $\left(v_{\max }, \mathrm{cm}^{-1}\right): 3387,3062$,
1584, 1529, 1480, 1241, 1100. ${ }^{1} \mathrm{HNMR}(400.13 \mathrm{MHz}$, $\left.\mathrm{CDCl}_{3}\right) \delta(\mathrm{ppm}):$ 7.26-7.78 (m, 14H), $9.40(\mathrm{~s}, 1 \mathrm{H})$. ${ }^{13} \mathrm{CNMR}\left(100.62 \mathrm{MHz}, \mathrm{CDCl}_{3}\right) \delta$ (ppm): 121.8, 125.6, 126.0, 126.7, 127.4, 127.7, 127.7, 128.1, 131.0.

2.2n Compound 5'c (table 1): m.p. $209-211^{\circ} \mathrm{C}$; Yield $0.281 \mathrm{~g}, 90 \%$; IR (KBr) $\left(v_{\max }, \mathrm{cm}^{-1}\right): 3450,3385$, 1584, 1529, 1480, 1240, 1098. ${ }^{1} \mathrm{HNMR}(400.13 \mathrm{MHz}$, $\left.\mathrm{CDCl}_{3}\right) \delta(\mathrm{ppm}): 6.92(\mathrm{~d}, J=7.28 \mathrm{~Hz}, 1 \mathrm{H}), 7.08(\mathrm{~d}$, $J=7.7 \mathrm{~Hz}, 1 \mathrm{H}), 7.26-7.62(\mathrm{~m}, 12 \mathrm{H}), 9.36(\mathrm{~s}, 1 \mathrm{H})$, $12.83(\mathrm{~s}, 1 \mathrm{H}) .{ }^{13} \mathrm{CNMR}\left(100.62 \mathrm{MHz}, \mathrm{CDCl}_{3}\right) \delta(\mathrm{ppm})$ : 111.3, 116.8, 117.9, 122.0, 126.3, 127.1, 127.3, 128.0, $129.5,144.6,156.4$.

2.2o Compound 5'd (table 1): m.p. $204-206^{\circ} \mathrm{C}$; Yield $0.300 \mathrm{~g}, 92 \%$; IR $(\mathrm{KBr})\left(v_{\max }, \mathrm{cm}^{-1}\right)$ : 3388, 3062, 2933, 1584, 1530, 1481, 1240, 1099. ${ }^{1} \mathrm{HNMR}$ (400.13 MHz, DMSO-d 6 ) $\delta$ (ppm): 4.05 (s, 3H), 7.03$7.69(\mathrm{~m}, 13 \mathrm{H}), 8.50(\mathrm{~d}, J=8 \mathrm{~Hz}, 1 \mathrm{H}), 10.51(\mathrm{~s}, 1 \mathrm{H})$. ${ }^{13} \mathrm{CNMR}$ (100.62 MHz, DMSO-d $\left.\mathrm{d}_{6}\right) \delta$ (ppm): 126.4, $126.8,127.3,128.2,128.5,129.0,129.6,129.9,130.2$, 131.0, 132.9, 133.9.

2.2p Compound 5'e (table 1): m.p. $227-230^{\circ} \mathrm{C}$; Yield $0.286 \mathrm{~g}, 88 \%$; IR (KBr) $\left(v_{\max }, \mathrm{cm}^{-1}\right): 3433,3027$, 1613, 1578, 1542, 1248, 1176. ${ }^{1} \mathrm{HNMR}(400.13 \mathrm{MHz}$, DMSO-d $\left.{ }_{6}\right) \delta(\mathrm{ppm}): 3.87(\mathrm{~s}, 3 \mathrm{H}), 6.98-7.86(\mathrm{~m}, 10 \mathrm{H})$, $7.55(\mathrm{~s}, 2 \mathrm{H}), 7.85(\mathrm{~d}, J=8.4 \mathrm{~Hz}, 2 \mathrm{H}), 9.23(\mathrm{~s}$, 1H). ${ }^{13}$ CNMR (100.62 MHz, DMSO-d 6 ) $\delta$ (ppm): 55.3, 114.2, 122.7, 126.7, 127.3, 127.8, 128.5, 146.1, 160.1 .

2.2q Compound 5'f (table 1): m.p. $235-236^{\circ} \mathrm{C}$; Yield $0.321 \mathrm{~g}, 80 \%$; IR $(\mathrm{KBr})\left(v_{\max }, \mathrm{cm}^{-1}\right)$ : 3054, 3018, 2930, 1608, 1578, 1541, 1230, 1181. ${ }^{1} \mathrm{HNMR}$ (400.13 MHZ, DMSO-d 6 ) $\delta(\mathrm{ppm}) 5.16(\mathrm{~s}, 2 \mathrm{H}), 7.11-$ $7.54(\mathrm{~m}, 17 \mathrm{H}), 8.01(\mathrm{~d}, J=8.8 \mathrm{~Hz}, 2 \mathrm{H}), 12.51(\mathrm{~s}$, 1H). ${ }^{13} \mathrm{CNMR}(100.62 \mathrm{MHz}$, DMSO-d 6 ) $\delta$ (ppm): 69.7, 115.4, 123.8, 126.0, 127.1, 127.5, 128.2, 128.3, 128.6, $128.8,128.9,129.1,135.7,137.4,146.0,158.9$.

2.2r Compound 5'g (table 1): m.p. $283-286^{\circ} \mathrm{C}$; Yield $0.372 \mathrm{~g}, 97 \%$; IR (KBr) $\left(v_{\max }, \mathrm{cm}^{-1}\right)$ : 3350, 3054, 2950, 1601, 1532, 1500. ${ }^{1} \mathrm{HNMR}(400.13 \mathrm{MHz}$, DMSO-d $\left._{6}\right) \delta(\mathrm{ppm}): 4.01(\mathrm{~s}, 2 \mathrm{H}), 7.33-8.00(\mathrm{~m}, 15 \mathrm{H})$, $8.130(\mathrm{~d}, J=7.6 \mathrm{~Hz}, 1 \mathrm{H}), 8.32(\mathrm{~s}, 1 \mathrm{H}), 12.733(\mathrm{~s}$, 1H). ${ }^{13}$ CNMR (100.62 MHz, DMSO-d $\left.\mathrm{d}_{6}\right) \delta$ (ppm): 39.9, 120.3, 120.6, 122.3, 124.5, 125.6, 126.7, 127.4, 128.2, $128.9,129.3,141.2,141.6,143.8,143.9,146.4 . \mathrm{m} / \mathrm{z}$ : $384 \mathrm{M}^{+}, 340,190,165,134,91,65$. Anal. Calcd for $\mathrm{C}_{28} \mathrm{H}_{20} \mathrm{~N}_{2}$ : C, 87.47; H, 5.24; N, 7.29. Found: C, 87.41; $\mathrm{H}, 5.29 ; \mathrm{N}, 7.22$. 
2.2s Compound $\mathbf{5}^{\prime} \mathbf{h}$ (table 1): m.p. $311-313^{\circ} \mathrm{C}$; Yield $0.294 \mathrm{~g}, 88 \%$; IR (KBr) $\left(v_{\max }, \mathrm{cm}^{-1}\right): 3413,3055$, 1598, 1490, 1451. ${ }^{1}$ HNMR (400.13 MHZ, DMSO-d 6 ) $\delta$ (ppm): 7.128-7.587 (m, 13H), $8.006(\mathrm{~d}, J=2.4 \mathrm{~Hz}$, $1 \mathrm{H}), 8.462(\mathrm{~d}, J=7.2 \mathrm{~Hz}, 1 \mathrm{H}), 11.404(\mathrm{~s}, 1 \mathrm{H}), 12.4$ (s, 1H). ${ }^{13} \mathrm{CNMR}(100.62 \mathrm{MHz}$, DMSO-d 6 ) $\delta(\mathrm{ppm})$ : $106.9,112.1,120.2,121.9,122.4,124.5,125.5,127.3$, 128.0, 128.9, 136.7, 144.1. m/z: $335 \mathrm{M}^{+}, 165,142$, 115, 77, 55. Anal. Calcd for $\mathrm{C}_{23} \mathrm{H}_{17} \mathrm{~N}_{3}$ : C, 82.36; $\mathrm{H}$, 5.11; N, 12.53. Found: C, 82.29; H, 5.21; N, 12.40.

2.2t Compound $\mathbf{5}^{\prime} \mathbf{i}$ (table 1): m.p. $257-259^{\circ} \mathrm{C}$; Yield $0.310 \mathrm{~g}, 94 \%$; IR (KBr) $\left(v_{\max }, \mathrm{cm}^{-1}\right): 3386,3062$, 1584, 1529, 1481, 1240, 1099. ${ }^{1} \mathrm{HNMR}$ (400.13 MHZ, $\left.\mathrm{CDCl}_{3}\right) \delta(\mathrm{ppm}): 6.99-7.36(\mathrm{~m}, 12 \mathrm{H}), 7.86(\mathrm{~d}, J=$ $7 \mathrm{~Hz}, 2 \mathrm{H}), 12.09$ (s, 1H). ${ }^{13} \mathrm{CNMR}(100.62 \mathrm{MHz}$, $\left.\mathrm{CDCl}_{3}\right) \delta(\mathrm{ppm}): 125.9,126.7,127.3,127.6,128.2$, 132.7, 144.3.

$2.2 \mathrm{u}$ Compound $\mathbf{5}^{\prime} \mathbf{j}$ (table 1): m.p. $308-309^{\circ} \mathrm{C}$; Yield $0.313 \mathrm{~g}, 92 \%$; IR (KBr) $\left(v_{\max }, \mathrm{cm}^{-1}\right): 3380,3065$, 1580, 1527, 1479, 1239, 1099, 810, 758. ${ }^{1} \mathrm{HNMR}$ (400.13 MHZ, DMSO-d $\left.\mathrm{d}_{6}\right) \delta$ (ppm): 7.30-7.53 (m, $10 \mathrm{H}), 7.78(\mathrm{t}, J=8 \mathrm{~Hz}, 1 \mathrm{H}), 8.51(\mathrm{~d}, J=8 \mathrm{~Hz}, 1 \mathrm{H})$, $8.95(\mathrm{t}, J=1.8 \mathrm{~Hz}, 1 \mathrm{H}), 9.41(\mathrm{~d}, J=8 \mathrm{~Hz}, 1 \mathrm{H}), 13.10$

Table 2. The effect of solvents in synthesis of polysubstituted imidazoles for model reaction.

\begin{tabular}{lccc}
\hline Entry & Solvent & Time (min) & Yield (\%) \\
\hline 1 & Water & 90 & 46 \\
2 & Ethanol & 35 & 75 \\
3 & Methanol & 40 & 78 \\
4 & Chloroform & 120 & 49 \\
5 & Acetonitrile & 80 & 73 \\
6 & Solvent-free & 30 & 96 \\
\hline
\end{tabular}

(s, 1H), ${ }^{13} \mathrm{CNMR}\left(100.62 \mathrm{MHz}, \mathrm{DMSO}-\mathrm{d}_{6}\right) \delta$ (ppm): 119.4, 122.6, 127.1, 128.4, 128.6, 130.4, 131.1, 131.8, $143.3,148.3$.

\section{Results and discussion}

Firstly, the synthesis of 1-benzyl 2,4,5-triphenyl imidazoles was chosen as a model reaction (compound 5a) in the synthesis of polysubstituted imidazoles to determine the optimum condition for these synthesises. In model reaction, in the presence of $\mathrm{SbCl}_{3}(5 \mathrm{~mol} \%)$ as catalyst, the mixture of benzil ( $1 \mathrm{mmol})$, benzaldehyde $(1 \mathrm{mmol})$, ammonium acetate $(1 \mathrm{mmol})$, bnzylamine $(1 \mathrm{mmol})$ carried out in different solvents such as water, ethanol, methanol, chloroform, acetonitrile and solvent-free conditions. From these experiments, it was clearly demonstrated that the solvent-free condition is the best condition to accomplish this synthesis (table 2). The same results were obtained when $\mathrm{SnCl}_{2} \cdot 2 \mathrm{H}_{2} \mathrm{O}$ was handled as catalyst.

We carried out the model reaction in the absence of catalyst at solvent-free condition and room-temperature for $24 \mathrm{~h}$, which lead to very poor yield $(12 \%)$ of product. In the absence of catalyst to enhance the yield of the desired product, temperature of the reaction was increased to $200^{\circ} \mathrm{C}$, but no appreciable increment in the product yield was observed. Therefore, we found that the presence of the catalytic amount of antimony trichloride (or stannous chloride dihydrate) and solventfree condition are the best conditions for this synthesis.

We also evaluated quantity of required catalyst in synthesis of imidazole derivatives for model reaction (compound 5a). It was found that maximum yield (96\%) obtained, when the reaction was loaded with $5 \mathrm{~mol} \%$ of $\mathrm{SbCl}_{3}$ or $20 \mathrm{~mol} \%$ of $\mathrm{SnCl}_{2} \cdot 2 \mathrm{H}_{2} \mathrm{O}$. Same result was obtained from trisubstituted imidazoles when compound $\mathbf{5}^{\prime} \mathbf{a}$ was chosen as model reaction (table 3 ).

Table 3. Optimization of molar ratio of the catalysts in synthesis of tri and tetrasubstituted imidazoles.

\begin{tabular}{|c|c|c|c|c|c|}
\hline $\mathrm{ShCl}_{2}$ & $\begin{array}{l}\text { Trisubstituted } \\
\text { imidazole }\end{array}$ & $\begin{array}{l}\text { Tetrasubstituted } \\
\text { imidazole }\end{array}$ & C & $\begin{array}{l}\text { Trisubstituted } \\
\text { imidazole }\end{array}$ & $\begin{array}{l}\text { Tetrasubstituted } \\
\text { imidazole }\end{array}$ \\
\hline$(\mathrm{mol} \%)$ & Time (min)/Yield (\%) & Time (min)/Yield (\%) & $(\mathrm{mol} \%)$ & Time (min)/Yield (\%) & Time $(\min ) /$ Yield $(\%)$ \\
\hline 1 & $60 / 65$ & $110 / 60$ & 2 & $90 / 65$ & $90 / 60$ \\
\hline 2 & $50 / 75$ & $40 / 70$ & 5 & $90 / 75$ & $60 / 65$ \\
\hline 5 & $15 / 95$ & $30 / 96$ & 10 & $65 / 80$ & $60 / 75$ \\
\hline 10 & $45 / 82$ & $35 / 78$ & 15 & $60 / 82$ & $50 / 80$ \\
\hline 15 & $65 / 80$ & $70 / 83$ & 20 & $50 / 90$ & $50 / 96$ \\
\hline 20 & $75 / 78$ & $75 / 85$ & 25 & $75 / 78$ & $75 / 85$ \\
\hline
\end{tabular}


In the following study on the model reactions, we have examined the reactions at various temperatures to find out the effect of temperature on the progress of reaction in the presence of optimized amount of catalysts (table 4). The maximum rate of reaction was obtained at $120^{\circ} \mathrm{C}$ in the presence of $\mathrm{SbCl}_{3}$ and at $140^{\circ} \mathrm{C}$ in the presence of $\mathrm{SnCl}_{2} \cdot 2 \mathrm{H}_{2} \mathrm{O}$ as the optimum temperature for tri and tetrasubstituted imidazoles.

As can be seen from table 4 , at $80^{\circ} \mathrm{C}$, reaction was completed slowly. Increasing temperature to $120^{\circ} \mathrm{C}$ (to

Table 4. Optimization of temperature for model reaction.

\begin{tabular}{|c|c|c|c|c|c|}
\hline Temn & $\begin{array}{l}\text { Trisubstituted } \\
\text { imidazole }^{\mathrm{a}}\end{array}$ & $\begin{array}{l}\text { Tetrasubstituted } \\
\text { imidazole }^{\mathrm{a}}\end{array}$ & & $\begin{array}{l}\text { Trisubstituted } \\
\text { imidazole }\end{array}$ & $\begin{array}{l}\text { Tetrasubstituted } \\
\text { imidazole }^{\mathrm{b}}\end{array}$ \\
\hline$\left({ }^{\circ} \mathrm{C}\right)^{\mathrm{a}}$ & Time (min)/Yield (\%) & Time (min)/Yield (\%) & $\left({ }^{\circ} \mathrm{C}\right)^{\mathrm{b}}$ & Time (min)/Yield (\%) & Time $(\min ) /$ Yield $(\%$ \\
\hline 80 & $90 / 80$ & $110 / 75$ & 80 & $100 / 70$ & $110 / 68$ \\
\hline 100 & $75 / 82$ & $90 / 85$ & 100 & $75 / 80$ & $90 / 75$ \\
\hline 120 & $50 / 90$ & $30 / 96$ & 120 & $60 / 82$ & $60 / 80$ \\
\hline 140 & $60 / 85$ & $40 / 90$ & 140 & $50 / 90$ & $50 / 96$ \\
\hline 160 & $100 / 65$ & $45 / 70$ & 160 & $80 / 70$ & $60 / 70$ \\
\hline
\end{tabular}

${ }^{\mathrm{a}} \mathrm{SbCl}_{3}$ as catalyst

${ }^{\mathrm{b}} \mathrm{SnCl}_{2} \cdot 2 \mathrm{H}_{2} \mathrm{O}$ as catalyst

Table 5. Comparison of the results for the synthesis of 1-benzyl 2,4,5-triphenyl imidazole (compound 5a) as a model reaction with other catalysts.

\begin{tabular}{|c|c|c|c|c|}
\hline Catalyst & $\operatorname{Mol}(\%)$ & Solvent/Temp. $\left({ }^{\circ} \mathrm{C}\right)$ & Time $(\min ) /$ Yield $(\%)$ & [Refs] \\
\hline $\mathrm{SbCl}_{3}$ & 5 & Solvent free/120 & $30 / 96$ & This work \\
\hline $\mathrm{SnCl}_{2} .2 \mathrm{H}_{2} \mathrm{O}$ & 20 & Solvent free $/ 140$ & $50 / 96$ & This work \\
\hline $\mathrm{I}_{2}$ & 10 & Solvent free/100 & $60 / 85$ & 33 \\
\hline$\left[\left(\mathrm{CH}_{2}\right)_{4} \mathrm{SO}_{3} \mathrm{HMIM}\right]\left[\mathrm{HSO}_{4}\right]^{\mathrm{a}}$ & 15 & Solvent free $/ 140$ & $120 / 90$ & 34 \\
\hline $\mathrm{TFA}^{\mathrm{b}}$ & 20 & Solvent free, MW $(150 \mathrm{~W})$ & $4 / 92$ & 35 \\
\hline $\mathrm{K}_{5} \mathrm{CoW}_{12} \mathrm{O}_{40} \cdot 3 \mathrm{H}_{2} \mathrm{O}^{\mathrm{c}}$ & 10 & Solvent free/140 & $120 / 90$ & 36 \\
\hline $\mathrm{SiO}_{2}{ }^{\mathrm{d}}$ & $2 \mathrm{~g}$ & Solvent free, MW & $8 / 87$ & 37 \\
\hline $\mathrm{SiO}_{2}^{\mathrm{d}}$ & 0.1 & $\mathrm{CH}_{2} \mathrm{Cl}_{2}$, Solar heat & $120 / 80$ & 38 \\
\hline $\mathrm{AlPO}_{4}$ & 1 & Solvent free/140 & $120 / 85$ & 15 \\
\hline $\mathrm{BF}_{3} \cdot \mathrm{SiO}_{2}^{\mathrm{e}}$ & 21 & Solvent free $/ 140$ & $120 / 80$ & 39 \\
\hline L-Proline & 15 & $\mathrm{MeOH} / 60$ & $510 / 86$ & 25 \\
\hline $\mathrm{InCl}_{3} .3 \mathrm{H}_{2} \mathrm{O}$ & 10 & $\mathrm{MeOH} /$ r.t. & $444 / 79$ & 40 \\
\hline $\mathrm{ZrCl}_{4}$ & 20 & $\mathrm{CH}_{3} \mathrm{CN} /$ r.t. & $60 / 86$ & 41 \\
\hline
\end{tabular}

a3-Methyl-1-(4-sulphonic acid)-butylimidazolium hydrogen sulphate

${ }^{\mathrm{b}}$ Trifluoroacetic acid

${ }^{\mathrm{c}}$ Potassium dodecatugstocobaltate trihydrate

${ }^{\mathrm{d}}$ Silica gel as acidic support

e Silica-supported boron trifluoride

Table 6. Reusability results of catalysts on the reaction process for the model reaction.

\begin{tabular}{|c|c|c|c|}
\hline \multirow[b]{2}{*}{ Product } & \multirow[b]{2}{*}{$\begin{array}{c}\text { Total } \\
\text { reusability }\end{array}$} & \multirow{2}{*}{$\begin{array}{c}\mathrm{SbCl}_{3} \\
\text { Yield (\%)/ } \\
\text { Time (min) }\end{array}$} & \multirow{2}{*}{$\begin{array}{c}\mathrm{SnCl}_{2} \cdot 2 \mathrm{H}_{2} \mathrm{O} \\
\text { Yield (\%)/ } \\
\text { Time (min) }\end{array}$} \\
\hline & & & \\
\hline & 1 & $96 / 30$ & $96 / 50$ \\
\hline & 2 & $95 / 30$ & $95 / 50$ \\
\hline & 3 & $95 / 35$ & $95 / 50$ \\
\hline & 4 & $97 / 45$ & $94 / 60$ \\
\hline & 5 & $97 / 55$ & $92 / 60$ \\
\hline
\end{tabular}




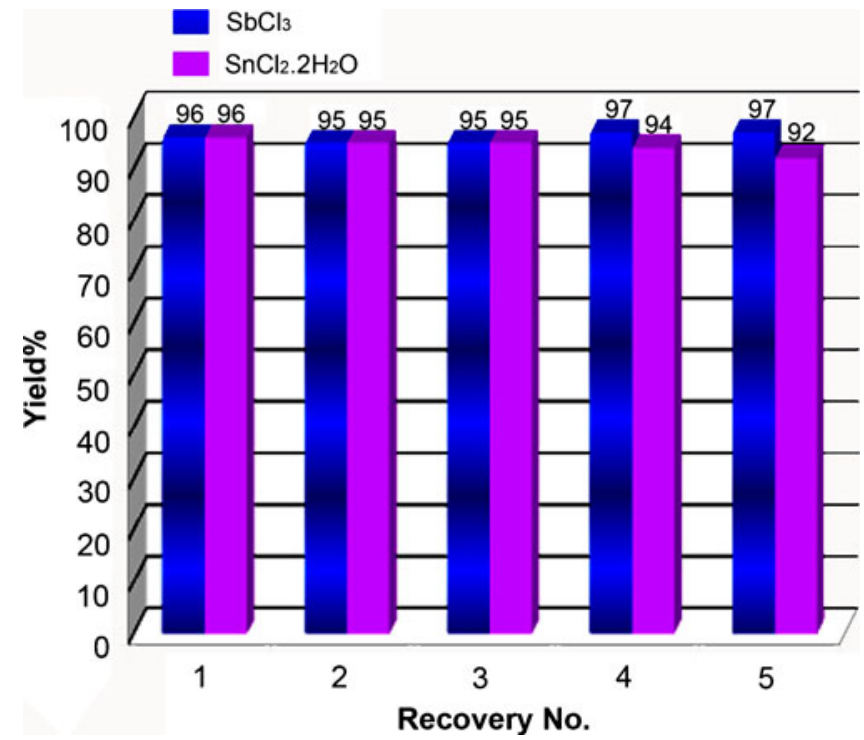

Figure 1. The reusability of the catalysts for model reaction.

$140^{\circ} \mathrm{C}$ for $\mathrm{SnCl}_{2} \cdot 2 \mathrm{H}_{2} \mathrm{O}$ ), increased the yield of reaction and decreased the time of reaction. When the reaction was heated above $120^{\circ} \mathrm{C}$ (above $140^{\circ} \mathrm{C}$ for $\mathrm{SnCl}_{2} \cdot 2 \mathrm{H}_{2} \mathrm{O}$ ), it was observed that high temperatures did not further improve the yield and did not decrease the reaction time.

According to the archived optimal condition, we conducted the synthesis of polysubstituted imidazoles in solvent-free condition at $120^{\circ} \mathrm{C}$ in the presence of $\mathrm{SbCl}_{3}$ and at $140^{\circ} \mathrm{C}$ in the presence of $\mathrm{SnCl}_{2} \cdot 2 \mathrm{H}_{2} \mathrm{O}$ as catalysts.
Comparison of this method with others for the synthesis of 1-benzyl 2,4,5-triphenyl imidazole (compound 5a) as a model reaction is shown in table $5 .^{15,25,33-41}$ These results show that these catalysts are in good conditions for the synthesis of imidazole derivatives than other catalysts and methods that were reported. This method not only affords the products in excellent yields, but also avoids the problems associated with catalyst cost, handling, safety, and pollution.

\subsection{Reusability of the catalyst}

At the end of the reactions, the catalysts were filtered, washed with diethyl ether, dried at $130^{\circ} \mathrm{C}$ for $1 \mathrm{~h}$, and reused in another reaction. We found that antimony trichloride and stannous chloride dihydrate showed high catalytic activity with very short reaction times. Moreover, can be recovered and reused five times without significant loss of activity. The results of these observations for the model reaction are shown in table 6 and figure 1.

A probable mechanism for the synthesis of tetrasubstituted imidazoles may be postulated as shown below (scheme 2).

As can be seen from scheme 2 , in the presence of catalyst as Lewis acid $\left(\mathrm{SbCl}_{3}\right.$ for example), the carbonyl group of aldehyde $\mathbf{2}$ is activated and the energy of transition state for nucleophilic attack is decreased. Then nucleophilic attack of amine $\mathbf{3}$ on the activated carbonyl

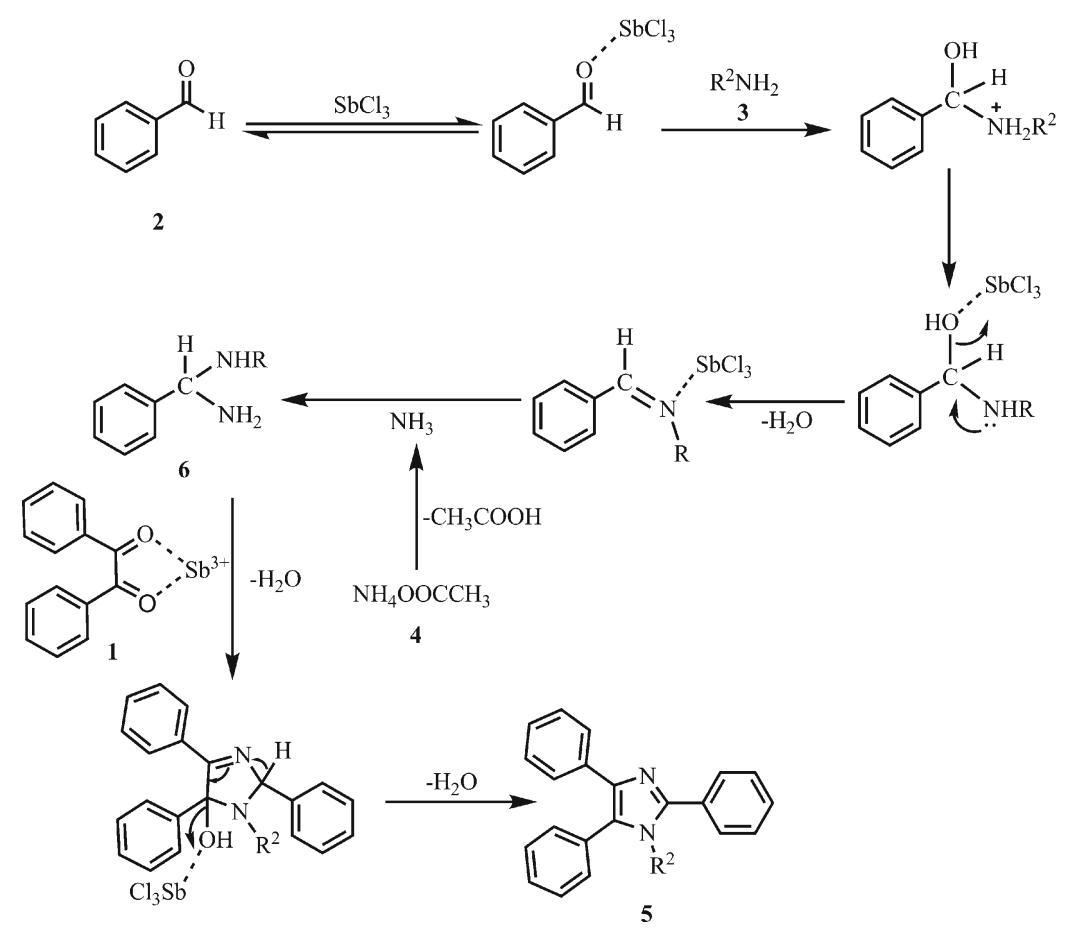

Scheme 2. The suggested mechanism for the synthesis of tetrasubstituted imidazoles. 
of aldehydes, resulted to the formation of imine, and it followed by nucleophilic attack of the in situ generated ammonia from 4 to the imine, giving the intermediate 6. From condensation of intermediate $\mathbf{6}$ with benzil $\mathbf{1}$ and dehydration of it, corresponding imidazoles $\mathbf{5}$ are produced.

Moreover, the probable mechanism for the synthesis of trisubstituted imidazoles is same with tetrasubstituted imidazoles but in this case, ammonium acetate was handled instead of primary amine. As well as, the reaction with aliphatic diketones and aliphatic aldehydes was tested, but the reaction progress was unsuccessful and no products were obtained. We believe that it is due to enolization of aliphatic diketones and aliphatic aldehydes which may cause reducing activity of aldehydes and 1,2-diketones mechanistically and no desired products were obtained.

\section{Conclusions}

In this work, we reported a thermal exposure synthetic method for the preparation of polysubstituted imidazoles. It is a simple, efficient and rapid via multicomponent one-pot reaction in the presence of $\mathrm{SbCl}_{3}$ and $\mathrm{SnCl}_{2} \cdot 2 \mathrm{H}_{2} \mathrm{O}$ as inexpensive and effective catalysts in solvent-free condition. Furthermore, products were isolated in excellent yields. Also, catalyst was efficiently recovered and reused, that is considered as economic advantages for the synthesis. It is believed that this procedure would find important applications in the synthesis of wide range of polysubstituted imidazoles.

\section{Acknowledgement}

We thank Yasouj University of Iran for financial support.

\section{References}

1. Weber L 2002 Curr. Med. Chem. 92085

2. a) Hulme C and Gore V 2003 Curr. Med. Chem. 10 51; b) Kantam M L, Mahendar K and Bhargava S $2010 \mathrm{~J}$. Chem. Sci. 122 63; c) Supale A R and Gokavi G S 2010 J. Chem. Sci. 122 189; d) Shanthi G and Perumal P T 2010 J. Chem. Sci. 122415

3. Domanska U and Kozlowska M K 2003 Fluid Phase Equilib. 206253

4. Isikdag I and Meric A 1999 Boll. Chim. Farm. 13824

5. Lee J C, Laydon J T, McDonnell P C, Gallagher T F, Kumar S, Green D, McNulty D, Blumenthal M J, Keys J R, Vatter S W L, Strickler J E, McLughlin M M, Siemens I R, Fisher S M, Livi G P, White J R, Adams J L and Young P R 1994 Nature 372739

6. Tackle A K, Brown M J B, Davies S, Dean D K, Francis G, Gaiba A, Hird A W, King F D, Lovell P J, Naylor A,
Reith A D, Steadman J G and Wilson D M 2006 Bioorg. Med. Chem. Lett. 16378

7. De Laszlo S E, Hacker C, Li B, Kim D, MacCoss M, Mantlo N, Pivnichny J V, Colwell L, Koch G E, Cascieri M A and Hagmenn W K 1999 Bioorg. Med. Chem. Lett. 9641

8. Schmierer R, Mildenberger $\mathrm{H}$ and Buerstell H German Patent 36146419871988 Chem. Abstr. 10837838

9. Wang L, Woods K W, Li Q, Barr K J, McCroskey R W, Hannick S M, Gkerke L, Credo R B, Hui Y H, Marsh K, Warener R, Lee J Y, Zielinsky-Mozng N, Frost D, Rosenberg S H and Sham H L 2002 J. Med. Chem. 45 1697

10. Maier T, Schmierer R, Bauer K, Bieringer H, Buerstell H and Sachse B US Patent 482033519891989 Chem. Abst. 111 19494w

11. a) Aridoss G, Sarca V D, Ponder J F Jr, Crowe J and Laali K K 2011 Org. Biomol. Chem. 9 2518; b) Sarca V D and Laali K K 2006 Green Chem. 8 615; c) Macedo A, Wendler E P, Dos Santos A A, Zukerman-Schpector J and Tiekinkc E R T 2010 J. Braz. Chem. Soc. 21 1563; d) Gay R M, Manarin F, Brandão R and Zeni G $2010 \mathrm{~J}$. Braz. Chem. Soc. 21 1635; e) Mandal S K, Paira M and Roy S C 2010 J. Chem. Sci. 122 423; f) Jing F, Zhang Y, Luo S, Chu W, Zhang H and Shi X 2010 J. Chem. Sci. 122 621; g) Ali A, Singh A P and Gupta R $2010 \mathrm{~J}$. Chem. Sci. 122 311; h) Pandit S S, Bhalerao S K, Aher U S, Adhav G L and Pandit V U 2011 J. Chem. Sci. 123 421

12. a) García J J, Zerecero-Silva P, Reyes-Rios G, Crestani M G, Arévalo A and Barrios-Francisco R 2011 Chem. Commun. 47 10121; b) Bellina F, Cauteruccio S, Di Fiore A, Marchetti C and Rossi R 2008 Tetrahedron 64 6060; c) Kamijo S and Yamamoto Y 2007 Chem. Asian J. 2568

13. Stoeck V and Schunack W 1974 Arch. Pharm. 307922

14. Hasaninejad A, Zare A, Shekouhy M and Ameri- Rad J 2010 J. Comb. Chem. 12844

15. Reddy P P, Mukkanti K and Purandar K 2010 Rasayan J. Chem. 3335

16. Davidson D, Weiss M and Jelling M 1937 J. Org. Chem. 2319

17. Stoeck V and Schunack W 1976 Arch. Pharm. 309421

18. Lipshutz B H and Morey M C 1983 J. Org. Chem. 48 3745

19. Ratnadeep S J, Priyanka G M, Sanjay K D and Charansingh H G 2010 J. Chin. Chem. Soc. 571227

20. a) Kong L, Lv X, Lin Q, Liu X, Zhou Y and Jia Y 2010 Org. Process Res. Dev. 14 902; b) Wolkenberg S E, Wisnoski D D, Leister W H, Wang Y, Zhao Z and Lindsley C W 2004 Org. Lett. 61453

21. a) Karami B, Hoseini S J, Eskandari K, Ghasemi A and Nasrabadi H 2012 Catal. Sci. Technol. 2 331; b) Karami B, Eskandari K and Khodabakhshi S 2012 Arkivoc 9 76; c) Karami B, Montazerozohori M and Habibi M H 2006 Phosphorus Sulfur Silicon Relat. Elem. 181 2825; d) Karami B, Montazerozohori M, Habibi M H and Zolfigol M A 2005 Heterocycl. Commun. 11513

22. Karimi A R, Alimohammadi Z and Amini M M 2010 Mol. Diversity 14635

23. Kantevari S S, Vuppalapati V N, Biradar D O and Nagarapu L 2007 J. Mol. Catal. A: Chem. 266109 
24. Blank J W, Durant G J, Emmett J C and Ganellin C R 1974 Nature 24865

25. Samai S, Chandra-Nandi G, Singh P and Singh M S 2009 Tetrahedron 6510155

26. Balalaie S and Arabanian A 2000 Green Chem. 2274

27. Shelke K F, Sapkal S B and Shingare M S 2009 Chin. Chem. Lett. 20283

28. Jain A K, Ravichandran V, Sisodiya M and Agrawal R K 2010 Asian Pac. J. Trop. Med. 3471

29. Shen M, Cai C and Yi W 2008 J. Fluorine Chem. 129 541

30. Khosropour A R 2008 Ultrason. Sonochem. 15659

31. Ahmad S and Abbas R 2006 J. Mol. Catal. A: Chem. 249246

32. Wang L M, Wang Y H, Tian H, Yao Y F, Shao J H and Liu B 2006 J. Fluorine Chem. 1271570

33. Ren Y M and Cai C 2010 J. Chem. Res. 34133
34. Davoodnia A, Heravi M M, Safavi-Rad Z and TavakoliHoseini N 2010 Synth. Commun. 402588

35. Mohammadizadeh M R, Hasaninejad A and Bahramzadeh M 2009 Synth. Commun. 393232

36. Nagarapu L, Apuri S and Kantevari S 2007 J. Mol. Catal. A: Chem. 266104

37. Balalaie S, Hashemi M M and Akhbari M 2003 Tetrahedron Lett. 441709

38. Mekheimer R A, Abdel Hameed A M, Mansour S A A and Sadek K U 2009 Chin. Chem. Lett. 20812

39. Sadeghi B, Mirjalili B B F and Hashemi M M 2008 Tetrahedron Lett. 492575

40. Sharma S D, Hazarika P and Konwar D 2008 Tetrahedron Lett. 492216

41. Sharma G V M, Jyothi Y and Lakshmi P S 2006 Synth. Commun. 362991 